\title{
Comparison of motor response to apomorphine and levodopa in Parkinson's disease
}

\author{
P A Kempster, J P Frankel, G M Stern, A J Lees
}

\begin{abstract}
The magnitude and pattern of motor responses to single doses of subcutaneous apomorphine and oral levodopa were compared in 14 patients with Parkinson's disease. Although apomorphine produced much shorter motor responses than levodopa, the quality of response to the two drugs was virtually indistinguishable. These clinical observations support the notion that integrity of striatal post-synaptic dopamine receptors is a key determinant of responsiveness to dopaminergic treatment in Parkinson's disease.
\end{abstract}

Many patients with Parkinson's disease develop unsatisfactory control of motor symptoms after prolonged treatment with levodopa. This may take the form of oscillating motor function, with periods of severe Parkinsonian disability alternating with periods of relative mobility marred by dyskinetic involuntary movements. Other patients show weaker responsiveness to levodopa and progressive deterioration of motor function without conspicuous fluctuations. Differences in the topography of motor deficits are apparent in some patients, and may be reflected in the pattern of responsiveness to levodopa and also in the pattern of levodopa induced involuntary movements. For example, severe gait freezing is resistant to levodopa in some patients although upper limb and cranial muscle function may show responsiveness, giving the impression of a patient who is "on" in the upper half of the body and "off" in the lower half of the body.
The neurochemical basis for such variations in clinical response to pharmacological treatment remains incompletely understood. Both pre-synaptic changes associated with capacity for surviving nigro-striatal terminals to synthesise, store and release dopamine formed from exogenous levodopa, ${ }^{1}$ and post-synaptic factors concerning preservation and sensitivity of striatal dopamine receptors may be of importance.

Apomorphine, a dopamine receptor agonist, has potent, rapid and therapeutically useful anti-Parkinsonian effects when injected subcutaneously. ${ }^{2-5}$ Apomorphine does not share transport or metabolic pathways with levodopa and probably acts by direct stimulation of striatal post-synaptic dopamine receptors. If, as has been suggested, responsiveness to levodopa depends on the metabolic and synaptic capacity of surviving pre-synaptic nigro-striatal terminals, one would expect to observe significant differences in the clinical effectiveness of the two agents in individual patients.

We have compared the magnitude and pattern of motor response to levodopa and apomorphine in 14 Parkinsonian patients using a variety of quantitative clinical parameters of motor function.

\section{Patients and methods}

Fourteen Parkinsonian patients (seven male, seven female) were studied. The mean age (range) was $57 \cdot 1$ years (43-68) and mean duration (range) of disease was $12 \cdot 2$ years $(4 \cdot 5-23)$. All patients were on chronic levodopa/ decarboxylase inhibitor medication, mean treatment duration (range) being $9 \cdot 8$ years

Table Summary of clinical details of all patients with modified Webster disability scale scores for levodopa and apomorphine studies. Severity of motor fluctuations: stable motor function $(S)$, moderate motor fluctuations $(+)$, severe oscillations $(++)$. Hoehn and Yahr grading refers to "off", state motor function

\begin{tabular}{|c|c|c|c|c|c|c|c|c|c|c|c|}
\hline \multirow{3}{*}{$\begin{array}{l}\text { Case } \\
\text { number }\end{array}$} & \multirow[b]{3}{*}{ Age/sex } & \multirow{3}{*}{$\begin{array}{l}\text { Disease } \\
\text { duration }\end{array}$} & \multirow{3}{*}{$\begin{array}{l}\text { Levodopa } \\
\text { duration }\end{array}$} & \multirow{3}{*}{$\begin{array}{l}\text { Hoehn and } \\
\text { Yahr }\end{array}$} & \multirow{3}{*}{$\begin{array}{l}\text { Motor } \\
\text { fluctuations }\end{array}$} & \multicolumn{4}{|c|}{ Modified Webster Score } & \multirow{2}{*}{\multicolumn{2}{|c|}{$\begin{array}{l}\text { Motor response } \\
\text { duration (minutes) }\end{array}$}} \\
\hline & & & & & & \multicolumn{2}{|c|}{ “off” } & \multicolumn{2}{|c|}{ "on" } & & \\
\hline & & & & & & apo & $l-d$ & apo & $l-d$ & apo & $l-d$ \\
\hline 1 & $62 /$ Female & 10 & 10 & 3 & ++ & 20 & 20 & 3 & 3 & 80 & 315 \\
\hline 2 & 43/Male & 5 & 5 & 4 & ++ & 20 & 20 & 5 & 5 & 47 & 250 \\
\hline 3 & $68 /$ Female & 9 & 7 & 4 & ++ & 25 & 25 & 10 & 11 & 45 & 175 \\
\hline 4 & $39 /$ Female & 4.5 & $2 \cdot 5$ & 3 & + & 17 & 17 & 2 & 2 & 55 & 150 \\
\hline 5 & 68/Male & 16 & 12 & 3 & ++ & 25 & 25 & 10 & 10 & 75 & 250 \\
\hline 6 & 60/Male & 16 & 16 & 3 & ++ & 22 & 24 & 6 & 6 & 35 & 140 \\
\hline 7 & 54/Male & 23 & 10 & 4 & ++ & 22 & 22 & 10 & 10 & 50 & 260 \\
\hline 8 & 48/Male & 15 & 13 & 3 & ++ & 17 & 17 & 7 & 7 & 70 & 265 \\
\hline 9 & 61/Male & 9 & 7 & 2 & $s$ & 11 & 11 & 2 & 2 & 70 & 210 \\
\hline 10 & $53 /$ Female & 14 & 14 & 3 & ++ & 24 & 24 & 5 & 5 & 65 & 205 \\
\hline 11 & $66 /$ Female & 7 & 4 & 3 & + & 21 & 23 & 7 & 6 & 65 & 150 \\
\hline 12 & 63/Female & 15 & 10 & 4 & $S$ & 16 & 17 & 8 & 8 & 45 & 180 \\
\hline 13 & 53/Male & 10 & 10 & 3 & + & 24 & 25 & 8 & 8 & 30 & 210 \\
\hline 14 & $62 /$ Female & 17 & 17 & 4 & ++ & 31 & 32 & 14 & 13 & 50 & 190 \\
\hline
\end{tabular}


(2.5-17). Parkinsonian disability ranged from moderate to severe and 12 patients had symptomatic fluctuations of motor function, these being severe in nine. Mean "off" phase Hoehn and Yahr stage (range) was 3.3 (2-4). Clinical details are summarised in table 1.

The studies were performed early in the morning after all anti-Parkinsonian medication had been withheld overnight. On one day, an oral dose of levodopa $250 \mathrm{mg} /$ carbidopa $25 \mathrm{mg}$ was administered and on a second day, a subcutaneous injection of apomorphine. Mean apomorphine dose was $2 \mathrm{mg}$ ( $1-4 \mathrm{mg}$ ), dose was determined in each case by doubling a previously established threshold dose for motor response. Studies were not necessarily performed on consecutive days. All patients were given domperidone $20 \mathrm{mg}$ by mouth two hours before the apomorphine injection and remained fasting for the duration of the levodopa and apomorphine studies.

Motor function was assessed at five to 15 minute intervals following levodopa or apomorphine administration and continued until after the motor response had worn off, as judged by pre-dose baseline motor scores. The following assessment was performed as described previously: ${ }^{6}$ unilateral hand tapping count over 30 seconds, timed walking over a twelve metre distance (failure to complete the task within 30 seconds was recorded as 30 seconds) and scoring of severity of tremor according to a four point scale.

Dyskinetic involuntary movements were scored on a similar four point scale for each of six anatomical zones (each limb, craniocervical, trunk) giving a maximum dyskinesia score of 24. In addition, patients were scored in pre-dose "off" phases and at time of peak motor response using a modified Webster disability scale ${ }^{6}$ evaluation of twelve domains of Parkinsonian disability with a maximum disability score of 36 . The amplitude of motor response was calculated for each motor parameter by subtracting pre-dose "off" motor score from the best score obtained during the subsequent "on" period.

Statistical significance was assessed by

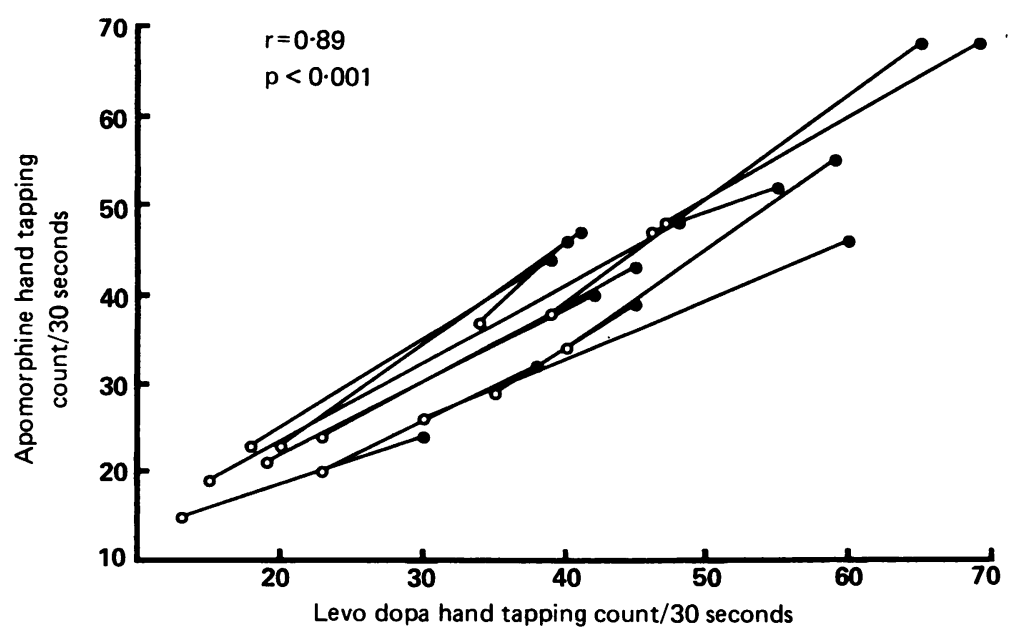

Figure 1 Correlation of pre-dose (open circles) and peak motor response (closed circles) hand tapping counts for apomorphine and levodopa.
Student's $t$ test with comparison of paired data. Linear correlation coefficients were calculated to examine amplitude of motor response and "on" period motor scores for levodopa and apomorphine.

\section{Results}

All parameters of motor function showed close correlation between apomorphine and levodopa for both amplitude of motor response and best "on" period motor function. The mean amplitude of hand tapping count response was 18 for apomorphine and 20 for levodopa $(r=0.92, p<0.001)$ and mean peak "on" phase tapping scores were 47 for apomorphine and 48 for levodopa $(r=0.89$, $\mathrm{p}<0.001$ ) (fig 1). Mean walking time response amplitude was $-12 \cdot 5 \mathrm{~s}$ for apomorphine and $-12.3 \mathrm{~s}$ for levodopa $(\mathrm{r}=0.81, \mathrm{p}<$ 0.001 ) and mean best "on" walking time was $12 \cdot 1 \mathrm{~s}$ for apomorphine and $11 \cdot 1 \mathrm{~s}$ for levodopa $(\mathrm{r}=0.80, \mathrm{p}<0.001)$. No patient had dyskinesia when "off" or tremor when "on", so amplitude and "on" scores for these motor parameters were the same. Mean apomorphine dyskinesia amplitude was 6.6 and mean levodopa dyskinesia amplitude was $7.0(\mathrm{r}=$ $0.92, \mathrm{p}<0.001)$. Mean apomorphine tremor amplitude was -0.9 and mean levodopa tremor amplitude was $-1 \cdot 1(\mathrm{r}=0.90, \mathrm{p}<$ $0.001)$. Mean modified Webster score amplitude was -14.2 for apomorphine and -14.7 for levodopa $(r=0.96, p<0.001)$ and mean "on" phase Webster score was 6.9 for both drugs $(r=0.98, p<0.001)$ (table 1$)$. There was no statistically significant difference in mean response amplitude or "on" phase scores between the two drugs for any motor parameter.

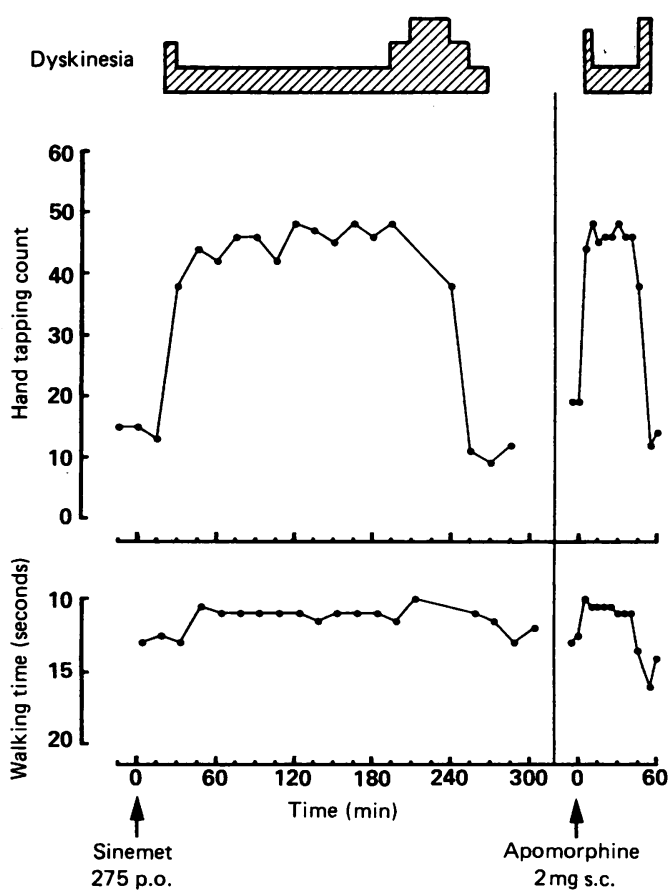

Figure 2 Serial hand tapping, walking and dyskinesia scores in Case 2 in relation to levodopa and apomorphine doses. Both drugs produce a similar biphasic pattern of dyskinetic involuntary movements. 
Not only was the overall magnitude of the response similar for apomorphine and levodopa, but both agents produced an almost identical pattern of response so far as the topography of motor deficits and dyskinesia in individual patients was concerned. The relative responsiveness of upper limbs (hand tapping) and gait function (walking time) in each case was the same for both drugs. One patient (Case 7) had a degree of drug resistant gait freezing which limited walking time improvement in "on" phases following both apomorphine and levodopa administration. Another patient (Case 2) usually had biphasic dyskinetic involuntary movements on oral levodopa medication. The single dose levodopa and apomorphine studies both showed a biphasic pattern of dyskinesia with similar topography and severity of involuntary movements.

Mean duration (range) of motor response to apomorphine injection was 56 minutes (30$80)$ compared with 211 minutes (140-315) for oral levodopa. Time to onset varied between three and 14 minutes (mean 7.9) for apomorphine and 19 and 75 minutes (mean 35.4) for levodopa. There was a weak correlation for duration of motor response for the two drugs $(\mathrm{r}=0.45, \mathrm{p}<0.5)$ and none for time to onset of response $(r=0 \cdot 16)$.

In 12 cases a transient deterioration in hand tapping performance to below pre-dose baseline level was observed following wearing off of motor response to both levodopa and apomorphine. Mean pre-dose to post-motor response tapping count ratio was 28 to 19 for levodopa ( $\mathrm{p}<0.01$ ) and 29 to 22 for apomorphine $(\mathrm{p}<0.001)$.

\section{Discussion}

The magnitude of clinical response and topography of response pattern to levodopa and apomorphine appear to be virtually identical in individual patients. Intensity, topography and time course of dyskinetic movements show similar parallels between the two agents, allowing for a shorter overall duration of motor response to apomorphine. Our results did not confirm specific effectiveness of apomorphine in reducing dyskinesia or Parkinsonian tremor. ${ }^{78} \mathrm{We}$ also observed transient worsening of the Parkinsonian state to below pre-dose level at the conclusion of motor responses. This phenomenon has previously been described for levodopa and may represent a transient inhibitory effect of low or declining plasma levodopa levels. ${ }^{9}$ Apomorphine and levodopa appear to have similar capacity to produce this response pattern in individual patients.

Animal experimental data suggests that apomorphine exerts its anti-Parkinsonian effects by direct interaction with striatal postsynaptic dopamine receptors. Apomorphine administration actually suppresses firing rate, and presumably synaptic dopamine release, in nigro-striatal neurons by stimulating pre-synaptic dopamine receptors. ${ }^{111}$ The equivalence of clinical response to apomorphine and levodopa argues against a fundamentally different mechanism of action for levodopa involving physiological restoration of synaptic dopaminergic neurotransmission by surviving nigro-striatal terminals. Were this true, it would be expected that differences in response amplitude between the two agents would be found, particularly in patients with advanced disease and more severe loss of nigral neurons. Our findings are more compatible with Melamed's proposal of a "non-physiological" action of levodopa. ${ }^{12} \mathrm{He}$ had suggested that exogenous levodopa may undergo biotransformation to dopamine at miscellaneous sites within the striatum, escape into the extracellular space and interact with postsynaptic dopamine receptors independently of synaptic activity in remaining nigro-striatal terminals. A corollary to this concept that levodopa and dopamine receptor agonist agents share similar striatal pharmacodynamics is that the capacity for individual patients to respond to dopamine receptor stimulating pharmacological treatment is determined by post-synaptic factors, especially striatal dopamine receptor status. While severity of nigral cell loss and hence striatal endogenous dopamine deficiency probably defines "off" state disability, it is proposed that "on" phase motor function and amplitude of motor response depends on preservation and sensitivity of striatal postsynaptic dopamine receptors, if essential nondopaminergic components of the extrapyramidal motor system are intact.

The practical importance of these studies concerns the assessment of the goals of pharmacological treatment in individual Parkinsonian patients. Amplitude of motor response to levodopa (or apomorphine) can be readily assessed by observing motor function in "off" and "on" states in relation to a single dose. Although some long phase variation in levodopa responsiveness can occur, such assessments indicate the best motor function attainable with drug therapy. Successful pharmacological manipulation will result in increased "on" state duration per day but will not substantially improve the quality of "on" states. Patients who respond only weakly to levodopa cannot be expected to show a greater response to other agents or other modes of administration. Similarly, aspects of Parkinsonian disability in individual patients, such as gait freezing, which do not respond to test doses of levodopa are likely to be resistant to other forms of dopaminergic drug treatment.

PAK and JPF were supported by the Kate Stillman Research Fellowship.

1 Hornykiewicz $\mathrm{O}$. The mechanisms of action of L-dopa in Parkinson's disease. Life Sci 1974;15:1249-59.

2 Stibe CMH, Lees AJ, Kempster PA, Stern GM. Subcutaneous apomorphine in Parkinsonian on-off oscillations. Lancet 1988;i:403-6.

3 Poewe W, Kleedorfer B, Gerstenbrand F, Oertel W. Subcutaneous apomorphine in Parkinson's disease. Lancet 1988;i:943.

4 Chaudhuri KR, Critchley P, Abbott RJ, Pye IF, Millac PAH. Subcutaneous apomorphine for on-off oscillations in Parkinson's disease. Lancet 1988;ii: 1260. 
5 Pollak P, Champay AS, Hommel M, Perret JE, Benabid AL. Subcutaneous apomorphine in Parkinson's disease. $J$ Neurol Neurosurg Psychiatry 1989;52:544.

6 Kempster PA, Frankel JP, Bovingdon M, Webster R, Lees AJ, Stern GM. Levodopa peripheral pharmacokinetics aj, Stern GM. Levodopa peripheral pharmacokinetics Neurol Neurosurg Psychiatry 1989;52:718-23.

7 Duby SE, Cotzias GC, Papavasilou PS, Lawrence WH. Injected apomorphine and orally administered levodopa in Parkinsonism. Arch Neurol 1972;27:474-80.

8 Braham J, Sarova-Pinhas I, Goldhammer V. Apomorphine in Parkinsonian tremor. Br Med J 1970;iii:768.

9 Nutt JG, Gancher ST, Woodward WR. Does an inhibitory action of levodopa contribute to motor fluctuations?
Neurology 1988;38:1553-7.

10 Bunney BS, Aghajanian GK, Roth RH. Comparison of effects of L-dopa, amphetamine and apomorphine on firing rate of rat dopaminergic neurons. Nature New Biology 1973;245:123-5.

11 Aghajanian GK, Bunney BS. Dopamine's autoreceptors: pharmacological characterization by microiontophoretic single cell recording studies. Naunyn-Schmiedebergs Arch

Pharmacol 1977;291:1-7.
12 Melamed E. Mechanism of action of exogenous L-dopa: Is it a physiological therapy for Parkinson's disease. In: Jankovic J, Tolosa E, eds. Parkinson's disease and movement disorders. Baltimore: Urban and Schwarzenberg, 1988 87-94. 